\title{
Robust Algorithms for Direction-Finding in the Presence of Model Errors
}

\author{
A. LEE SWINDLEHURST \\ Electrical and Computer Engineering \\ Brigham Young University \\ Provo, Utah $8 \$ 602$
}

\begin{abstract}
The application of high-resolution, subspace-based methods to narrowband direction-of-arrival (DOA) estimation relies on several critical assumptions. Two of these are 1) that the response of the antenna array is known in all directions of interest, and 2) that the spatial covariance of the background noise is known. Neither of these assumptions is satisfied in practice, often resulting in a serious degradation of algorithm performance. While there has recently been much work directed at determining the asymptotic effects of the finite sample approximation, relatively little work has focussed on algorithm sensitivity to the above model errors. In this paper, a model error sensitivity analysis is carried out for some popular high-resolution subspace-based algorithms. Theoretical expressions for the covariance of the DOA estimation error are developed and compared with that obtained by simulation. The analysis is also used to develop optimally weighted versions of the algorithms that are robust to the types of model errors considered.
\end{abstract}

\section{INTRODUCTION}

$\mathbf{H}^{\prime}$

GH-RESOLUTION subspace-based methods for narrowband direction-of-arrival (DOA) estimation have been extensively studied during the past ten years. These methods can achieve performance far superior to classical techniques because they fully exploit the underlying data model, including knowledge of the antenna array response and spatial noise statistics. Nearly all of the analytical performance analyses that have been conducted for these algorithms have focussed on the asymptotic effects of using a finite noisy data set to estimate the DOAs. This is, however, only one of the many possible sources of estimation error. In many applications, the limiting factor in performance is not due to finite sample effects, but rather to errors in the model for the array response and spatial noise covariance.

While techniques have been proposed to mitigate the effects of these model errors $[1,2,3,4,5,6]$, little work has focused on obtaining analytical expressions for the perturbation induced in the DOA estimates. The special case of array calibration errors has recently been considered in $[7,8,9,10]$. In this paper, a sensitivity analysis is conducted for both array and noise model perturbations, and applied to MUSIC [11], (deterministic) maximum-likelihood (ML) [12], Cadzow's method (sometimes referred to as multidimensional MUSIC) [13], and weighted subspace fitting (WSF) $[14,15]$. This analysis is used to develop an optimal weighted version of MUSIC and optimal WSF-like algorithms that are robust against simple Gaussian array and noise perturbations.

\section{Subspace Based Algorithms}

To introduce notation, the unperturbed data model assumed for the narrowband DOA estimation problem is briefly described. Assume an m-element array of sensors, $d$ narrowband far-field

1 This work was supported in part by the SDIO/IST Program managed by the Office of Naval Research under Contract N0001485-K-0550, by the Joint Services Program at Stanford University (US Army, US Navy, US Air Force) under Contract DAAL03-88C-0011, by grants from Rockwell International and the General Electric Company, and by the IR\&D Program at ESL, Inc.

TH0331-9/90/0000-0362\$01.00"1990 IHEE emitters, and define $a(\theta) \in \mathbb{C}^{m}$ to be the array response for a narrowband emitter at DOA $\theta$. The array manifold is defined to be the set $\mathcal{A}=\{\mathbf{a}(\theta): \theta \in \Theta\}$ for some region $\theta$ in DOA space. The set $\mathcal{A}$ is assumed to be known, either analytically or via some calibration procedure, and to be unambiguous (i.e., any collection of $d \leq m$ distinct vectors from $\mathcal{A}$ is a linearly independent set). The output $z \in \mathcal{C}^{m}$ of the array at time $t$ is given by

$$
\mathbf{z}(t)=\mathbf{A}\left(\theta_{0}\right) \mathbf{s}(t)+\mathbf{n}(t)
$$

where $s(t) \in \mathbb{C}^{d}$ is the amplitude and phase of the signals at time $t, \mathbf{n}(t)$ is additive noise, $\theta_{0}=\left[\theta_{1}, \cdots, \theta_{d}\right]^{T}$, and

$$
\mathbf{A}\left(\theta_{0}\right)=\left[\mathbf{a}\left(\theta_{1}\right) \cdots \mathbf{a}\left(\theta_{d}\right)\right]
$$

Assuming spatially white noise that is uncorrelated with the signals, the array covariance is given by

$$
\mathbf{R}=\mathcal{E}\left\{\mathbf{z}(t) \mathbf{z}^{*}(t)\right\}=\mathbf{A}\left(\boldsymbol{\theta}_{0}\right) \mathbf{S} \mathbf{A}^{*}\left(\boldsymbol{\theta}_{0}\right)+\sigma^{2} \mathbf{I},
$$

where $\mathcal{E}$ denotes expectation, - denotes hermitian transpose, $S$ is the covariance of the emitter signals, and $\sigma^{2}$ is the noise variance at each sensor. The matrix $\mathbf{S}$ is assumed to be full rank, al though this assumption is not critical for some of the algorithms discussed. The eigendecomposition of $\mathbf{R}$ has the following form:

$$
\mathbf{R}=\sum_{i=1}^{m} \lambda_{i} \mathbf{e}_{i} \mathbf{e}_{i}^{*}=\mathbf{E}_{s} \mathbf{\Lambda}_{s} \mathbf{E}_{s}^{*}+\sigma^{2} \mathbf{E}_{n} \mathbf{E}_{n}^{*}
$$

where $\mathbf{E}_{d}=\left[\begin{array}{lll}\mathbf{e}_{1} & \cdots & \mathbf{e}_{d}\end{array}\right], \mathbf{E}_{n}=\left[\begin{array}{lll}\mathbf{e}_{d+1} & \cdots & \mathbf{e}_{m}\end{array}\right], \boldsymbol{\Lambda}_{s}=$ $\operatorname{diag}\left\{\lambda_{1} \cdots \lambda_{d}\right\}$, and $\lambda_{1} \geq \cdots \geq \lambda_{d}>\lambda_{d+1}=\cdots=\lambda_{m}=\sigma^{2}$. The span of the $d$ signal subspace eigenvectors $\mathbf{E}_{\text {s }}$ will be identical to that of $\mathbf{A}\left(\theta_{0}\right)$, so a full rank $d \times d$ matrix $\mathbf{T}$ will exist satisfying

$$
E_{s}=\mathbf{A}\left(\boldsymbol{\theta}_{0}\right) \mathbf{T} \text {. }
$$

The noise subspace eigenvectors $\mathbf{E}_{n}$ satisfy $\mathbf{E}_{n}^{*} \mathbf{A}\left(\theta_{0}\right)$.

The algorithms under consideration in this paper use $\mathbf{R}, \mathbf{E}_{s}$, and $\boldsymbol{E}_{n}$ to determine $\boldsymbol{\theta}_{0}$ as follows:

$$
\begin{aligned}
& \min _{\theta} V_{M U}=\min _{\theta} \frac{\mathbf{a}^{*}(\theta) \mathbf{E}_{n} \mathbf{E}_{n}^{*} \mathbf{a}(\theta)}{\mathbf{a}^{*}(\theta) \mathbf{a}(\theta)} \\
& \min _{\boldsymbol{\theta}} V_{M L}=\min _{\boldsymbol{\theta}} \operatorname{Tr}\left(\mathbf{P} \frac{1}{A} \mathbf{R}\right) \\
& \min _{\boldsymbol{\theta}, \mathbf{T}} V_{M D}=\min _{\boldsymbol{\theta}, \mathbf{T}}\left\|\mathbf{E}_{\theta}-\mathbf{A}(\theta) \mathbf{T}\right\|_{F}^{2} \\
& \min _{\boldsymbol{\theta}, \mathbf{T}} V_{W S F}=\min _{\boldsymbol{\theta}, \mathbf{T}}\left\|\mathbf{E}_{s} \hat{\mathbf{\Lambda}}_{s}^{-1 / 2}-\mathbf{A}(\theta) \mathbf{T}\right\|_{F}^{2}
\end{aligned}
$$

where $\mathbf{P}_{\hat{A}}=\mathbf{A}(\theta)\left(\mathbf{A}^{\bullet}(\theta) \mathbf{A}(\theta)\right)^{-1} \mathbf{A}^{*}(\theta), \bar{\Lambda}=\mathbf{A}_{s}-\sigma^{2} \mathbf{I}$, and $M U$, $M L, M D$, and $W S F$ refer respectively to MUSIC, maximum likelihood, MD-MUSIC, and weighted subspace fitting. Note that while MUSIC performs a one-dimensional search (i.e., the DOA estimates are obtained one at a time), the other algorithms are multidimensional (i.e., all DOAs are obtained simultaneously). It 
can be shown [16] that, for this analysis, all of the multidimensional methods can be considered to be special cases of the following subspace fitting minimization problem $[14,15]$ :

$$
\min _{\theta} V=\min _{\theta} \operatorname{Tr}\left(\mathbf{P}_{A}^{\perp} \mathbf{E}, \mathbf{W E} \mathbf{E}_{s}^{*}\right) \text {, }
$$

where the value of $\mathbf{W}$ corresponding to each algorithm is shown in Table 1 (it is shown in Section III.B how the weighting for the ML method is derived). The matrix $W$ can be interpreted as a

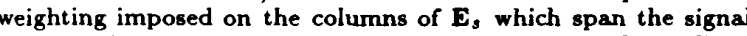
subspace (hence the term weighted subspace fitting in $[14,15]$ ).

\begin{tabular}{|c|c|}
\hline ALGORTTHM & WEIGHTING (W) \\
\hline ML & $\overline{\boldsymbol{\Lambda}}$ \\
\hline MD-MUSIC & $\mathbf{I}$ \\
\hline WSF & $\tilde{\mathbf{\Lambda}}^{2} \mathbf{\Lambda}_{s}^{-1}$ \\
\hline
\end{tabular}

Table 1: Summary of Subspace Fitting Algorithms

With perfect data, all of the above algorithms would of course produce the correct result. However, due to various unavoidable errors, the "true" covariance matrix and its eigendecomposition are not available, but rather some perturbed set $\hat{\mathbf{R}}, \hat{\mathbf{E}}_{\text {s }}$ and $\dot{\mathbf{E}}_{n}$. Consequently, the algorithms must use these perturbed quantities to compute an estimate $\hat{\theta}$ of $\theta_{0}$, and the magnitude of the error $\dot{\theta}=\dot{\theta}-\theta_{0}$ will be different for each method. In this paper, the error covariance $\mathbf{C}=\mathcal{E}\left\{\dot{\boldsymbol{\theta}} \tilde{\theta}^{*}\right\}$ will be analytically evaluated and used to judge the relative performance of the algorithms when the error is due to array and noise model perturbations.

\section{A First Order Error Analysis}

To isolate the effects of array and noise model errors on the DOA estimates, it will be assumed that the finite sample effects due to additive noise are negligible and that an exact measurement of the perturbed covariance $\mathbf{R}$ is available. A very general model for $\hat{\mathbf{R}}$ is as follows:

$$
\dot{\mathbf{R}}=(\mathbf{A}+\overline{\mathbf{A}}) \mathbf{S}(\mathbf{A}+\overline{\mathbf{A}})^{*}+\sigma^{2}(\mathbf{I}+\overline{\mathbf{\Sigma}}),
$$

where $\dot{\mathbf{A}}$ and $\dot{\boldsymbol{\Sigma}}$ are the result of errors in the array and noise models, respectively. The matrix $\dot{A}$ incorporates into $\hat{\mathbf{A}}$ the effects of imprecisely known sensor locations, perturbations in the antenna amplitude and phase patterns, and mutual coupling effects. Deviation of the noise covariance matrix from its nominal value is denoted by the hermitian matrix $\tilde{\boldsymbol{\Sigma}}$.

\section{A. The Perturbed Eigendecomposition}

Since the algorithms under consideration are based on properties of the eigenvectors of $\mathbf{R}$, of primarily interest are the effects of $\overline{\mathbf{A}}$ and $\overline{\mathbf{E}}$ on $\mathbf{E}$, and $\mathbf{E}_{n}$. Let $\overline{\mathbf{E}}_{s}=\overline{\mathbf{E}}_{g}+\overline{\mathbf{E}}_{\text {, and }} \hat{\mathbf{E}}_{n}=\mathbf{E}_{n}+\tilde{\mathbf{E}}_{n}$ represent the perturbed signal and noise subspaces, respectively. Since, in general, $\hat{\boldsymbol{E}}_{n}$ is unique and $\boldsymbol{E}_{n}$ is not (it may be replaced by $\mathbf{E}_{n} \Phi$ for any full-rank $\Phi$ ), care must be taken in using the latter equation. For purposes of this analysis, it will be assumed that $\dot{\mathbf{E}}_{n}^{*} \dot{\mathbf{E}}_{n}=\mathbf{I}$ and that $\mathbf{E}_{n}$ and $\dot{\mathbf{E}}_{n}$ are approximately orthogonal.

To establish a link between the model errors of (4) and the subspace errors $\overline{\mathbf{E}}_{s}$ and $\overline{\mathbf{E}}_{n}$, the eigenvector equations

$$
\begin{aligned}
& \hat{\mathbf{R}} \hat{\mathbf{E}}_{s}=\hat{\mathbf{E}}_{0}\left(\boldsymbol{\Lambda}_{0}+\tilde{\boldsymbol{\Lambda}}_{0}\right) \\
& \hat{\mathbf{R}} \hat{\mathbf{E}}_{n}=\dot{\mathbf{E}}_{n}\left(\sigma^{2} \mathbf{I}+\dot{\boldsymbol{\Lambda}}_{n}\right)
\end{aligned}
$$

will be examined, where $\bar{\Lambda}_{\text {s }}$ and $\bar{\Lambda}_{n}$ represent the perturbed signal and noise eigenvalues, respectively. Expanding equation (5) using the model of (4) and eliminating second-order error terms (e.g., terms of order $O\left(\|\overline{\mathbf{A}}\|^{2}\right), O(\|\overline{\mathbf{A}}\| \cdot\|\dot{\mathbf{E}}\|$,$) , etc.) leads to the following$ first-order approximation:

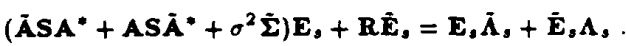

After multiplying on the left by $P \frac{1}{A}$, a few simple algebraic manipulations yield the following:

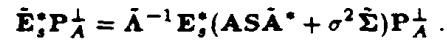

A similar relationship may be obtained for the noise eigenvectors using equation (6). Eliminating second-order error terms from (6) and multiplying on the left by $\mathbf{P}_{A}$ leads to

$$
\dot{\mathbf{E}}_{n}^{*} \mathbf{A}=-\mathbf{E}_{n}^{*}\left(\overline{\mathbf{A}}+\sigma^{2} \overline{\mathbf{\Sigma}} \mathbf{A}^{\dagger \cdot \mathbf{S}^{-1}}\right) .
$$

Equations (7) and (8) are the key relationships used below in developing error expressions for the DOA estimates.

\section{B. General Error Expressions}

In $[8,10,16,17]$, the following approximate expressions for the DOA estimation error are developed using a simple first-order analysis for MUSIC:

$$
\begin{aligned}
\left(\hat{\theta}-\theta_{0}\right)_{M U} & \simeq-\left[V_{M U}^{\prime \prime}\left(\theta_{0}\right)\right]^{-1} \dot{V}_{M U}^{\prime}\left(\theta_{0}\right) \\
\hat{V}_{M U, i}^{\prime}\left(\theta_{0}\right) & \simeq 2 \operatorname{Re}\left[\mathrm{d}^{*}\left(\theta_{i}\right) \mathbf{E}_{n} \dot{\mathbf{E}}_{n}^{*} \mathbf{a}\left(\theta_{1}\right)\right] \\
V_{M U}^{\prime \prime}\left(\theta_{0}\right) & =-2 \operatorname{Re}\left[\left(\mathbf{D}^{*} \mathbf{P}_{A}^{1} \mathbf{D}\right) \odot \mathbf{I}\right]
\end{aligned}
$$

and the subspace fitting methods defined by equation (3):

$$
\begin{aligned}
\left(\hat{\theta}-\theta_{0}\right)_{S F} & \simeq-\left[V^{\prime \prime}\left(\theta_{0}\right)\right]^{-1} \hat{V}^{\prime}\left(\theta_{0}\right) \\
\hat{V}_{1}^{\prime}\left(\theta_{0}\right) & \simeq 2 \operatorname{Re}\left[\operatorname{Tr}\left(\mathbf{P}_{A}^{+} \mathbf{A}_{i} \mathbf{A}^{\dagger} \mathbf{E}, \mathbf{W} \tilde{E}_{s}^{*}\right)\right] \\
V^{\prime \prime}\left(\theta_{0}\right) & =-2 \operatorname{Re}\left[\left(\mathbf{D}^{*} \mathbf{P}_{A}^{+} \mathbf{D}\right) \odot\left(\mathbf{A}^{\dagger} \mathbf{E}_{s} \mathbf{W} \mathbf{E}_{\xi}^{*} \mathbf{A}^{\dagger *}\right)^{T}\right]
\end{aligned}
$$

where

$$
\begin{aligned}
& \hat{V}^{\prime}\left(\theta_{0}\right)=\left.\frac{\partial \hat{V}}{\partial \theta}\right|_{\theta=\theta_{0}} \\
& =\left[\hat{V}_{1}^{\prime}\left(\theta_{0}\right) \cdots \hat{V}_{d}^{\prime}\left(\theta_{0}\right)\right]^{T} \\
& V^{\prime \prime}\left(\theta_{0}\right)=\left.\frac{\partial^{2} V}{\partial \theta \partial \theta^{T}}\right|_{\theta=\theta_{0}} \\
& \mathbf{d}\left(\theta_{i}\right)=\left.\frac{\partial \mathbf{a}(\theta)}{\partial \theta}\right|_{\theta=\theta_{i}} \\
& \mathbf{D}=\left[\mathbf{d}\left(\theta_{1}\right) \cdots \mathbf{d}\left(\theta_{d}\right)\right]
\end{aligned}
$$

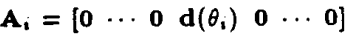

$$
\begin{aligned}
& \mathbf{A}^{\dagger}=\left(\mathbf{A}^{*} \mathbf{A}\right)^{-1} \mathbf{A}^{*}
\end{aligned}
$$

and $\hat{V}$ represents $V$ evaluated using $\hat{\mathbf{E}}$, or $\hat{\mathbf{E}}_{n}$ instead of $\mathbf{E}$, or $\mathbf{E}_{n}$. To link the error in the DOA estimates to the model perturbations, equations (7) and (8) are substituted into (10) and (9) to obtain

$$
\hat{V}_{M U, i}^{\prime}\left(\theta_{0}\right) \simeq 2 \operatorname{Re}\left\{d^{*}\left(\theta_{i}\right) P_{A}^{\perp}\left[\bar{a}\left(\theta_{i}\right)+\sigma^{2} \overline{\bar{\Sigma}} \mathbf{A}^{\dagger \cdot} S_{i}^{-1}\right]\right\}
$$

and

$$
\hat{V}_{i}^{\prime}\left(\theta_{0}\right) \simeq 2 \operatorname{Re}\left\{\operatorname{Tr}\left[\mathbf{P} \frac{1}{A} \mathbf{A}_{i} \mathbf{A}^{\dagger} \mathbf{E}_{s} \mathbf{W} \tilde{\mathbf{\Lambda}}^{-1} \mathbf{E}_{s}^{*}\left(\mathbf{A S} \dot{\mathbf{A}}^{*}+\sigma^{2} \tilde{\boldsymbol{\Sigma}}\right)\right]\right\}
$$

where $S_{i}^{-1}$ is the $i^{\text {th }}$ column of $S^{-1}$. Note that for the deterministic ML method, a first-order approximation of the gradient

$$
\begin{aligned}
\hat{V}_{M L, i}^{\prime}\left(\theta_{0}\right) & =2 \operatorname{Re}\left\{\operatorname{Tr}\left[\mathbf{P}_{A}^{\perp} \mathbf{A}_{i} \mathbf{A}^{\dagger} \dot{\mathbf{R}}\right]\right\} \\
& \simeq 2 \operatorname{Re}\left\{\operatorname{Tr}\left[\mathbf{P}_{A}^{\perp} \mathbf{A}_{i} \mathbf{A}^{\dagger}\left(\mathbf{A S} \dot{A}^{*}+\sigma^{2} \dot{\Sigma}\right)\right]\right\},
\end{aligned}
$$


leads to precisely what would be obtained from equation (12) if $\mathbf{W}=\tilde{\Lambda}$. Thus, as mentioned eariler, the ML algorithm can be assumed for this analysis to be a special case of the general subspace fitting problem described in equation (3) and Table 1.

Given any particular deterministic perturbation $\tilde{\mathbf{A}}$ and $\tilde{\boldsymbol{\Sigma}}$, the DOA error $\boldsymbol{\theta}-\boldsymbol{\theta}_{0}$ could of course be evaluated directly without using the approximations given above. However, a more useful approach would be to assume that $\overline{\mathbf{A}}$ and $\overline{\boldsymbol{\Sigma}}$ are not known precisely, but are rather realizations of some known perturbation model. In this approach, one is not interested in a particular value of the estimation error $\overline{\boldsymbol{\theta}}$, but rather some averaged measure of it. In this paper, the perturbation model will be assumed to be given in probabilistic terms (i.e., $\overline{\mathbf{A}}$ and $\tilde{\boldsymbol{\Sigma}}$ are random), and expressions for the bias and variance of $\overline{\boldsymbol{\theta}}$ will be obtained. Assuming $\dot{\boldsymbol{A}}$ and $\dot{\Sigma}$ are zero-mean, it is clear that $\mathcal{E}\left(\hat{\theta}-\theta_{0}\right)=\mathbf{0}$ for all of the algorithms. The second-order statistics of the error are given by

$$
\begin{aligned}
\mathbf{C}_{M U} & =\left(V_{M U}^{\prime \prime}\right)^{-1} \mathbf{Q}_{M U}\left(V_{M U}^{\prime \prime}\right)^{-1} \\
\mathbf{C}_{S F} & =\left(V^{\prime \prime}\right)^{-1} \mathbf{Q}_{S F}\left(V^{\prime \prime}\right)^{-1}
\end{aligned}
$$

where

$$
\begin{aligned}
\mathbf{Q}_{M U} & =\mathcal{E}\left\{\left(\hat{V}_{M U}^{\prime}\right)\left(\hat{V}_{M U}^{\prime}\right)^{T}\right\} \\
\mathbf{Q}_{S F} & =\mathcal{E}\left\{\left(\hat{V}^{\prime}\right)\left(\hat{V}^{\prime}\right)^{T}\right\} .
\end{aligned}
$$

Thus, the error covariances can be computed for any scenario where $\mathbf{Q}_{M U}$ and $\mathbf{Q}_{S F}$ may be evaluated. This will in turn be possible provided that the second order statistics of the elements of $\overline{\mathbf{A}}$ and $\overline{\boldsymbol{\Sigma}}$ are known.

\section{A Simple Perturbation Model}

There are a variety of models that could be used to describe $\tilde{\mathbf{A}}$ and $\tilde{\boldsymbol{\Sigma}}$. A particularly simple model for $\overline{\mathbf{A}}$ is to assume that its columns are independent zero-mean complex Gaussian random vectors with a known angle-independent covariance:

$$
\tilde{\mathbf{a}}\left(\theta_{i}\right) \sim \mathscr{C} \mathcal{N}(0, \mathbf{B}), \mathcal{E}\left(\overline{\mathbf{a}}\left(\theta_{i}\right) \overline{\mathbf{a}}^{T}\left(\theta_{i}\right)\right)=0, i=1, \cdots, d .
$$

If the calibration errors are independent from sensor-to-sensor, $\mathbf{B}$ is clearly diagonal. Off-diagonal terms indicate sensor-to-sensor correlations that result, for example, if there are uncalibrated mutual coupling effects, or if some sensors tend to perturb uniformly (such as identical or adjacent elements).

With a few simple exceptions, it is somewhat more difficult than in the array error case to connect a particular model for $\bar{\Sigma}$ with some underlying physical phenomenon. A simple yet reasonable model for purposes of this paper is to assume that the elements of $\dot{\boldsymbol{\Sigma}}$ are independent, zero-mean, Gaussian random variables of equal variance $\sigma_{g}^{2}$. Under this model, the off-diagonal elements are assumed to be complex with independent real and imaginary parts of equal variance $\sigma_{s}^{2} / 2$, while the diagonal elements are assumed to be real.

Though the analysis of this paper is not restricted to the above error models, for the sake of brevity these will be the only two cases considered (several more general error models are described in $[16,17])$. These examples nonetheless provide a benchmark for determining algorithm sensitivity and making performance comparisons. Since it will always be assumed that $\boldsymbol{A}$ and $\dot{\boldsymbol{\Sigma}}$ are independent of one another, the covariance of the error for each model will be presented separately. In the general case where $\dot{\boldsymbol{A}}$ and $\dot{\boldsymbol{\Sigma}}$ are both non-zero, the error covariance will be given by the sum of the covariances due to each error source considered separately.

Since expressions for $V^{\prime \prime}$ and $V_{M U}^{\prime \prime}$ are already available from equations (9) and (10), the covariances $C_{M U}$ and $C_{S F}$ may be evaluated once the expectations $Q_{M U}$ and $Q_{S F}$ have been derived. For the array error model of (15), it can be shown that

$$
\begin{aligned}
\mathbf{Q}_{M U}=2 \operatorname{Re}\left[\left(\mathbf{D}^{*} \mathbf{P}_{A}^{\frac{1}{A}} \mathbf{B} \mathbf{P}_{A}^{\frac{1}{A}} \mathbf{D}\right) \odot \mathbf{I}\right] \\
\mathbf{Q}_{S F}=2 \operatorname{Re}\left[\left(\mathbf{D}^{*} \mathbf{P}_{A}^{\frac{1}{A}} \mathbf{B} \mathbf{P}_{A}^{\perp} \mathbf{D}\right) \odot\right. \\
\left.\left(\mathbf{A}^{\dagger} \mathbf{E}_{s} \mathbf{W} \mathbf{T}^{*} \mathbf{T} \mathbf{W}^{*} \mathbf{E}_{s}^{*} \mathbf{A}^{\dagger *}\right)^{T}\right],
\end{aligned}
$$

where $\mathbf{T}$ is as defined in equation (2). The corresponding expressions for the simple $\overline{\mathbf{Z}}$ model are

$$
\begin{aligned}
\mathbf{Q}_{M U}=2 \sigma_{s}^{2} \sigma^{4} \operatorname{Re}\left[\left(\mathbf{D}^{*} \mathbf{P}_{\mathbf{A}}^{\frac{1}{1}} \mathbf{D}\right) \odot\left(\mathbf{A}^{*} \mathbf{E}_{s} \tilde{\mathbf{A}}^{-2} \mathbf{E}_{s}^{*} \mathbf{A}\right)^{T}\right] \\
\mathbf{Q}_{S F}=2 \sigma_{s}^{2} \sigma^{4} \operatorname{Re}\left[\left(\mathbf{D}^{*} \mathbf{P} \frac{1}{A} \mathbf{D}\right) \odot\right. \\
\left.\left(\mathbf{A}^{\dagger} \mathbf{E}, \mathbf{W} \tilde{\mathbf{A}}^{-2} \mathbf{W}^{*} \mathbf{E}_{s}^{*} \mathbf{A}^{* *}\right)^{T}\right] .
\end{aligned}
$$

The performance of each of the subspace fitting algorithms encom passed by equation (3) may be ascertained by plugging into $V^{\prime \prime}$ and $Q_{S F}$ the appropriate value of $\mathbf{W}$ from Table 1 .

\section{Optimally Weighted Algorithms}

An advantage of the simple matrix expressions obtained for $\mathbf{C}_{M U}$ and $\mathbf{C}_{S F}$ in the previous section is that performance comparisons between different implementations of the algorithms are facilitated. In this section, for example, it will be demonstrated how optimal weightings may be derived to improve the quality of the DOA estimates under a particular perturbation model. The following weighted versions of MUSIC and subspace fitting will be considered:

$$
\begin{aligned}
\min _{\boldsymbol{\theta}} V_{W M U} & =\min _{\boldsymbol{\theta}} \frac{\mathbf{a}^{*}\left(\theta_{i}\right) \mathbf{E}_{\boldsymbol{n}} \mathbf{W}_{\mathrm{m}} \mathbf{E}_{n}^{*} \mathbf{a}\left(\theta_{i}\right)}{\mathbf{a}^{*}\left(\theta_{i}\right) \mathbf{a}\left(\theta_{i}\right)} \\
\min _{\boldsymbol{\theta}, \mathbf{T}} V_{R S F} & =\min _{\boldsymbol{\theta}, \mathbf{T}}\left\|\mathbf{W}_{r}^{1 / 2}\left(\mathbf{E}_{s} \mathbf{W}_{c}^{1 / 2}-\mathbf{A}(\theta) \mathbf{T}\right)\right\|_{F}^{2} \\
& =\min _{\boldsymbol{\theta}} \operatorname{Tr}\left(\mathbf{P}_{A^{\prime}}^{\perp}, \mathbf{E}^{\prime}, \mathbf{W}_{c} \mathbf{E}^{\prime *}\right)
\end{aligned}
$$

where $\mathbf{W}_{m}, \mathbf{W}_{c}$, and $\mathbf{W}_{r}$ are all hermitian and positive definite and where $\mathbf{A}^{\prime}$ and $\mathbf{E}^{\prime}$, denote the row-weighted matrices $\mathbf{W}_{r}^{1 / 2} \mathbf{A}$ and $\mathbf{W}_{r}^{1 / 2} \mathbf{E}$. All row-weighted quantities below will also be indicated by this notation.

Using the results of the previous section, it is straightforward to derive expressions for the error covariances $C_{W M U}$ and $C_{R S F}$. The second derivatives of the weighted algorithms are given by

$$
\begin{aligned}
& V_{W M U}^{\prime \prime}\left(\theta_{0}\right)=-2 \operatorname{Re}\left[\left(\mathbf{D}^{*} \mathbf{E}_{n} \mathbf{W}_{m} \mathbf{E}_{n}^{*} \mathbf{D}\right) \odot \mathbf{I}\right] \\
& V_{R S F}^{\prime \prime}\left(\theta_{0}\right)=-2 \operatorname{Re}\left[\left(\mathbf{D}^{\prime *} \mathbf{P}_{A^{\prime}}^{\perp} \mathbf{D}^{\prime}\right) \odot\left(\mathbf{A}^{\prime \dagger} \mathbf{E}_{s}^{\prime} \mathbf{W}_{c} \mathbf{E}_{s}^{\prime *} \mathbf{A}^{\prime \dagger *}\right)^{T}\right](19)
\end{aligned}
$$

With the following slightly modified version of equation (7),

$$
\dot{\mathbf{E}}_{g}^{\prime *} \mathbf{P}_{\mathbf{A}^{\prime}}^{\perp}=\tilde{\mathbf{A}}^{-1} \mathbf{E}_{\mathbf{g}}^{*}\left(\mathbf{A} \mathbf{S} \tilde{\mathbf{A}}^{\prime *}+\sigma^{2} \tilde{\mathbf{\Sigma}}\right) \mathbf{P}_{\mathbf{A}^{\prime}}^{\perp} \text {, }
$$

expressions for $\mathbf{Q}_{R S F}$ may be derived as before. For the array error model of (15),

$$
\begin{aligned}
\mathbf{Q W M U}_{W}=2 \operatorname{Re}\left[\left(\mathbf{D}^{*} \mathbf{E}_{n} \mathbf{W}_{m} \mathbf{E}_{n}^{*} \mathbf{B} \mathbf{E}_{n} \mathbf{W}_{m} \mathbf{E}_{n}^{*} \mathbf{D}\right) \odot \mathbf{I}\right] \\
\mathbf{Q}_{R S F}=2 \operatorname{Re}\left[\left(\mathbf{D}^{\prime *} \mathbf{P}_{A^{\prime}}^{\perp} \mathbf{W}_{r}^{1 / 2} \mathbf{B} \mathbf{W}_{\mathbf{r}}^{* / 2} \mathbf{P}_{A^{\prime}}^{\perp} \mathbf{D}^{\prime}\right) \odot\right. \\
\left.\left(\mathbf{A}^{\prime \dagger} \mathbf{E}^{\prime}, \mathbf{W}_{c} \mathbf{T}^{*} \mathbf{T} \mathbf{W}_{c}^{*} \mathbf{E}_{s}^{\prime *} \mathbf{A}^{\prime \dagger *}\right)^{T}\right]
\end{aligned}
$$

Using the error model for $\overline{\mathbf{\Sigma}}$ described earlier leads to

$$
\begin{aligned}
& \mathbf{Q}_{R S F}=2 \sigma_{s}^{2} \sigma^{4} \operatorname{Re}[\left(\mathbf{D}^{\prime *} \mathbf{P}_{\mathbf{A}^{\prime}}^{\perp}, \mathbf{W}_{\boldsymbol{r}} \mathbf{P}_{\mathbf{A}^{\prime}}^{\perp} \mathbf{D}^{\prime}\right) \odot \\
&\left.\left(\mathbf{A}^{\prime \prime} \mathbf{E}_{s}^{\prime} \mathbf{W}_{c} \tilde{\mathbf{\Lambda}}^{-2} \mathbf{W}_{c}^{*} \mathbf{E}_{s}^{\prime *} \mathbf{A}^{\prime \dagger *}\right)^{T}\right]
\end{aligned}
$$

Only an expression for $Q_{R S F}$ is given in this case since an optimal weighting will only be possible for the multidimensional subspace fitting class of algorithms.

Careful study of equations (13), (14), and (18) through (25) reveals that particular choices of $\mathbf{W}_{m}, \mathbf{W}_{c}$, and $\mathbf{W}_{r}$ will lead 364 
and $V_{R S F}^{\prime \prime}=Q_{R S F}$. For the array error case, the weights which produce this effect are

$$
\begin{aligned}
& \mathbf{W}_{m}=\left(\mathbf{E}_{n}^{*} \mathbf{B} \mathbf{E}_{n}\right)^{-1} \\
& \mathbf{W}_{c}=\left(\mathbf{T}^{*} \mathbf{T}\right)^{-1} \\
& \mathbf{W}_{r}=\mathbf{B}^{-1}
\end{aligned}
$$

while for noise model errors, the corresponding weights are

$$
\begin{aligned}
& \mathbf{W}_{c}=\dot{\boldsymbol{\Lambda}}^{2} \\
& \mathbf{W}_{r}=\mathbf{I} .
\end{aligned}
$$

It can be shown $[16,17]$ that these choices lead to optimal versions of the algorithms which achieve parameter estimates of minimum variance. In other words, for any admissible choice of $\mathbf{W}_{m}, \mathbf{W}_{c}$, and $\mathbf{W}_{\boldsymbol{r}}$,

$$
\begin{gathered}
\mathbf{C}_{W M U}\left(\left(\mathbf{E}_{n}^{*} \mathbf{B} \mathbf{E}_{n}\right)^{-1}\right) \leq \mathbf{C}_{W M U}\left(\mathbf{W}_{m}\right) \\
\mathbf{C}_{R S F}\left(\left(\mathbf{T}^{*} \mathbf{T}\right)^{-1}, \mathbf{B}^{-1}\right) \leq \mathbf{C}_{R S F}\left(\mathbf{W}_{c}, \mathbf{W}_{r}\right)
\end{gathered}
$$

for the array error case, and

$$
\mathrm{C}_{R S F}\left(\bar{\Lambda}^{2}, \mathbf{I}\right) \leq \mathbf{C}_{R S F}\left(\mathbf{W}_{c}, \mathbf{W}_{r}\right)
$$

for the noise error model. Note that since $\mathrm{C}_{W M U}(\mathrm{I})=\mathrm{C}_{M U}$ and $C_{R S F}(I, I)=C_{S F}$, the weighted versions of the algorithms will outperform their unweighted counterparts when the model errors are of the types described. Even though the optimal weights are a function of the unknown quantities $\mathbf{E}_{n}, \mathbf{T}$, and $\dot{\boldsymbol{\Lambda}}$, using the eigenvectors $\hat{\mathbf{E}}_{n}$ of the sample covariance and the estimates

$$
\begin{aligned}
& \hat{\boldsymbol{T}}=\mathbf{A}^{\dagger}(\hat{\boldsymbol{\theta}}) \hat{\mathbf{E}}, \\
& \hat{\boldsymbol{\Lambda}}=\hat{\boldsymbol{\Lambda}}_{s}-\dot{\sigma}^{2} \mathbf{I}
\end{aligned}
$$

only introduces variance terms of second-order and higher. Thus, to first order, optimal performance can achieved using the estimated weights $\mathbf{W}_{m}$ and $\hat{W}_{c}$.

An interesting consequence of the above results for the array error model of $(15)$ is that

$$
C_{W M U}\left(\left(E_{n}^{*} B E_{n}\right)^{-1}\right)=C_{R S F}\left(\left(T^{*} T\right)^{-1}, B^{-1}\right),
$$

and hence that the one-dimensional weighted MUSIC algorithm is no more sensitive to array errors of the type described by (15) than any of the multidimensional subspace fitting algorithms. In fact, if $\mathbf{B}=\gamma^{2} \mathbf{I}$, the above analysis demonstrates that MUSIC has lower estimate variance than MD-MUSIC, WSF, and the deterministic maximum likelihood method; i.e., for any $\mathbf{W}_{c}$ and $\mathbf{W}_{r}$,

$$
\mathbf{C}_{M U}=\left(\gamma^{2} / 2\right)\left\{\operatorname{Re}\left[\left(\mathbf{D}^{*} \mathbf{P} \frac{1}{A} \mathbf{D}\right) \odot \mathbf{I}\right]\right\}^{-1} \leq \mathbf{C}_{R S F}\left(\mathbf{W}_{c}, \mathbf{W}_{r}\right) \text {. }
$$

\section{Simulation EXamples}

In order to isolate the effects of the various model errors, finite sample effects are neglected by considering in each of the experiments in this section the exact perturbed covariance $\hat{\mathbf{R}}$. A total of 1000 trials were conducted for each example, with $\hat{\mathbf{R}}$ generated for each trial using the error-free covariance $\mathbf{R}$ and the distribution of the perturbation. The sample RMS error of the DOA estimates was then calculated and compared to that predicted by the corresponding theoretical expressions. In all of the following examples, the nominal gain of all sensors was assumed to be unity in the direction of the impinging signals, and it was assumed that the number of emitters $d$ had been correctly determined. In addition, the noise was assumed to be spatially white with unit variance, except for the case where noise covariance perturbations were studied.

\section{Case 1: Array Model Errors - Column Weighting}

For this case, a 10 element uniform linear array (ULA) with $\lambda / 2$ inter-element spacing was assumed. Two emitters were simulated, one at $0^{\circ}$ broadside and the other at $7^{\circ}$. The signal-to-noise ratio (SNR) for the $7^{\circ}$ source was $20 \mathrm{~dB}$, while that of the broadside source was varied between 0 and $12 \mathrm{~dB}$, and the two sources were $90 \%$ correlated with $0^{\circ}$ correlation phase. The covariance $B$ of (15) was set at $(0.01)^{2} I$, which corresponds to a $-40 \mathrm{~dB}$ gain error and a $0.57^{\circ}$ phase error standard deviation. Figure 1 shows a plot of the standard deviation of the $0^{\circ}$ source estimates for MD-MUSIC, deterministic ML, WSF, and the optimal subspace weighting. The connected lines indicate the theoretical predictions and the symbols represent the results of the simulations. Note the excellent agreement between the predicted and measured DOA errors, and also the improvement achieved by the optimal weighting $\mathbf{W}_{c}=\left(\hat{\mathbf{T}}^{*} \hat{\mathbf{T}}\right)^{-1}$ relative to $\mathrm{WSF}$ and deterministic ML (in this case, $\mathbf{W}_{r}=\mathbf{I}$ ). Though not shown on the plot, the onedimensional MUSIC algorithm has virtually identical performance to that of MD-MUSIC and the optimal weighting.

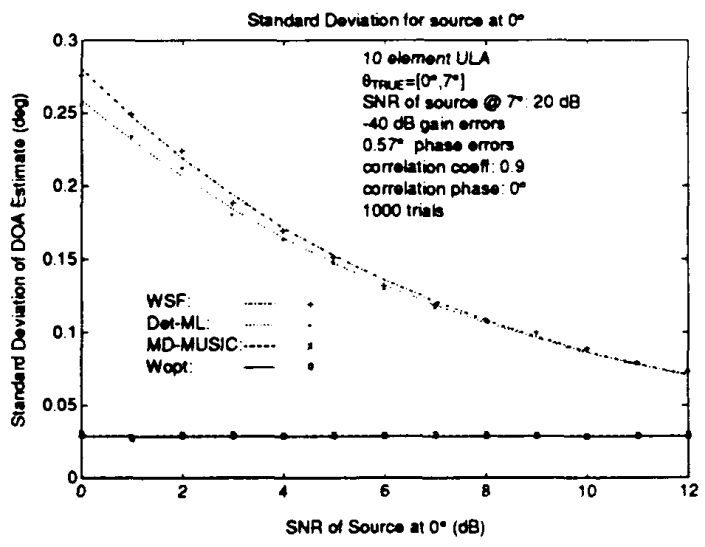

Figure 1: Actual and Predicted DOA Errors vs. SNR - Case 1

\section{Case 2: MUSIC and Unequal Array Errors}

For the next example, consider the array of Figure 2 which is composed of a seven element ULA and two additional elements separated from either end of the ULA by six wavelengths. Suppose that the error covariance $B$ is given by

$$
\mathbf{B}=\operatorname{diag}\left\{\left[10^{-2}, 10^{-4}, 10^{-4}, \cdots, 10^{-4}, 10^{-2}\right]\right\},
$$

so that the standard deviation of the array perturbation for the end elements is ten times greater than for the elements of the ULA. For this array, a relevant system design problem would be how to appropriately trade-off (a) the performance improvement which results from using the large aperture provided by the end elements, and (b) the performance degradation caused by the unreliable calibration information for these elements.

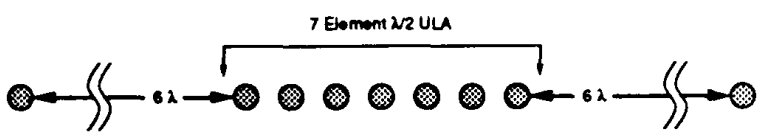

Figure 2: Linear Array for Case 2

The array of Figure 2 was simulated with the perturbation described by (28). Two uncorrelated emitters were assumed, each of power $0 \mathrm{~dB}$ relative to the additive noise. The first emitter was fixed at $0^{\circ}$ broadside, while the second was varied from $2^{\circ}$ to $50^{\circ}$ over several experiments. The dotted curve and the symbol $o$ represent the predicted and measured performance of MUSIC when using only the 7 element ULA and ignoring the end elements (no MUSIC result is shown for the case where the second source 
was at $2^{\circ}$ since the algorithm failed to resolve the two sources in over half the trials). The solid curve and the symbol * denote the predicted and measured performance of MUSIC for the full array (including the end elements) without weighting, while the dashed line and + denote the same for MUSIC with the weighting $\mathbf{W}_{m}=\left(\mathbf{E}_{n}^{*} \mathbf{B} \mathbf{E}_{n}\right)^{-1}$.

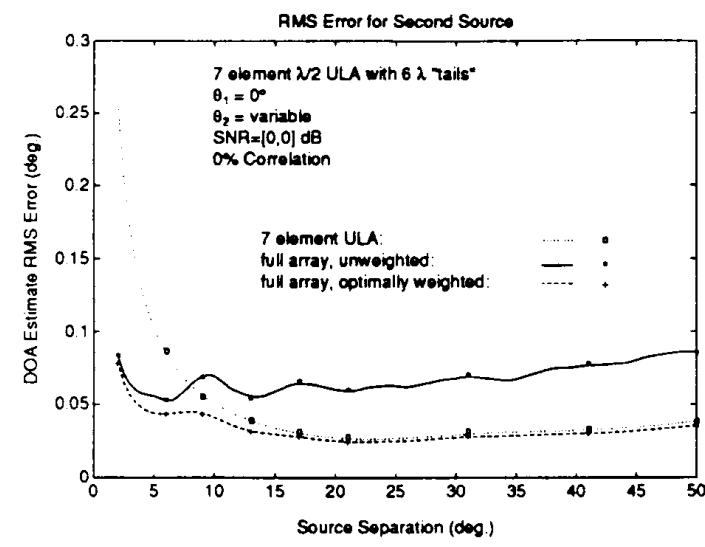

Figure 9: Actual and Predicted DOA Errors vs. $\theta_{2}$ - Case 2

When the sources are closely spaced, the smaller ULA does not provide enough aperture for MUSIC to accurately estimate the DOAs. As the second source is moved away from broadside, the performance of the ULA improves until at $\theta_{2} \simeq 8^{\circ}$ it does as well as the full unweighted array. For DOAs beyond $8^{\circ}$, using the information from the unweighted end elements actually degrades algorithm performance relative to just ignoring them. However, the lowest estimation error is achieved using the full optimally weighted array.

\section{Case 3: Error in Noise Covariance Model}

This example illustrates the effects of errors in the model for the noise covariance and demonstrates the advantage of using the optimal column weighting $\mathbf{W}_{\text {opt }}=\bar{\Lambda}^{2}$. A 12 element $\lambda / 2$ spaced ULA and two correlated sources at $10^{\circ}$ and $15^{\circ}$ were simulated. The SNR for each source was $0 \mathrm{~dB}$, and the standard deviation of the additive noise covariance perturbation was $\sigma_{9}=0.01$. The actual and predicted RMS error performance of MUSIC, WSF, deterministic ML, and the optimally weighted algorithm for the source at $15^{\circ}$ are plotted versus signal correlation in Figure 4 . Note that while the sensitivity of MUSIC increases dramatically at higher levels of correlation, the multidimensional methods remain relatively unaffected. In fact, the performance of WSF and $W_{\text {opt }}$ actually improves as the signals become more highly correlated. As predicted, the lowest RMS error is achieved by the subspace fitting algorithm with optimal column weighting.

\section{REFERENCES}

[1] A. Paulraj and T. Kailath, "Direction-of-Arrival Estimation by Eigenstructure Methods with Unknown Sensor Gain and Phase", In Proc. IEEE ICASSP, pages 17.7.1-17.7.4, Tampa, Fla., March 1985.

[2] A. Paulraj and T. Kailath, "Eigenstructure Methods for Direction of Arrival Estimation in the Presence of Unknown Noise Fields", IEEE Trans. ASSP, 34:13-20, February 1986.

(3] A. Paulraj, V. U. Reddy, T. J. Shan, and T. Kailath, "A Subspace Approach to Determine Sensor Gain and Phase with Applications to Array Processing", In Proc. $30^{\text {th }}$ SPIE International Technical Symposium, Advanced Algorithms and Architectures for Signal Processing, San Diego, CA., August 1986.

[4] B. Friedlander and A. J. Weiss, "Eigenstructure Methods for Direction Finding with Sensor Gain and Phase Uncertainties", In Proc. ICASSP '88, pages 2681-2684, 1988.

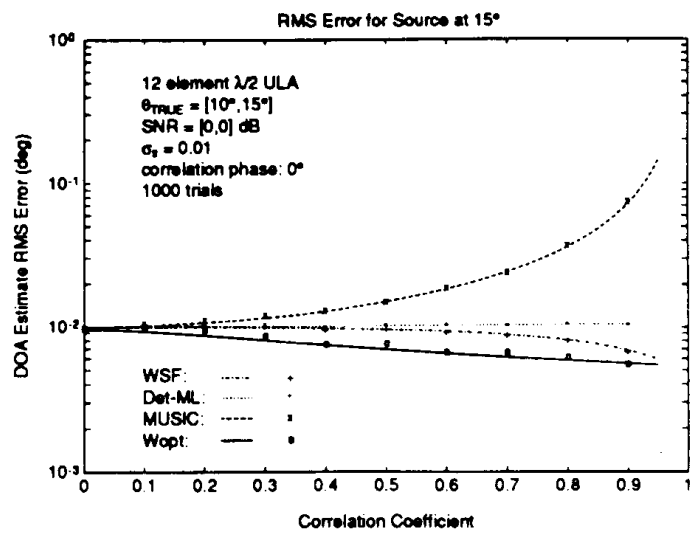

Figure 4: Actual and Predicted DOA Errors - Case 3

[5] A. J. Weiss, A. S. Willsky, and B. C. Levy, "Eigenstructure Approach for Array Processing with Unknown Intensity Coefficients", IEEE Trans. ASSP, 36:1613-1617, October 1988.

[6] A. J. Weiss and B. Friedlander, "Direction Finding in the Presence of Mutual Coupling", In Proc. 22nd A silomar Conf. on Signals, Systems, and Computers, pages 598-602, 1988.

[7] B. Friedlander, "A Sensitivity Analysis of the MUSIC Algorithm", In Proc. IEEE ICASSP, Glasgow, Scotland, May 1989.

[8] A. Swindlehurst, B. Ottersten, and T. Kailath, "An Analysis of MUSIC and Root-MUSIC in the Presence of Sensor Perturbations", In Proc. 23 ${ }^{\text {rd }}$ Asilomar Conference of Signals, Systems, and Computers, pages 930-934, Asilomar. CA., November 1989.

[9] B. Friedlander, "Sensitivity of the Maximum Likelihood Direction Finding Algorithm", In Proc. $23^{\text {rd }}$ Asilomar Con. ference of Signals, Systems, and Computers, pages 594-598, Asilomar, CA., November 1989.

[10] A. Swindlehurst and T. Kailath, "An Analysis of Subspace Fitting Algorithms in the Presence of Sensor Errors", In Proc. IEEE ICASSP, volume 5, pages 2647-2650, Albuquerque, NM, 1990.

[11] R. Schmidt, A Signal Subspace Approach to Multiple Emitter Location and Spectral Estimation, PhD thesis, Stanford University, 1981.

[12] M. Wax, Detection and Estimation of Superimposed Signals, PhD thesis, Stanford University, Stanford, CA., 1985.

[13] J. A. Cadzow, "A High Resolution Direction-of-Arrival Algorithm for Narrow-band Coherent and Incoherent Sources", IEEE Trans. on ASSP, 36(7):965-979, July 1988.

[14] B. Ottersten and M. Viberg, "Analysis of Subspace Fitting Based Methods for Sensor Array Processing", In Proc. IEEE JCA SSP, Glasgow, Scotland, May 1989.

[15] M. Viberg and B. Ottersten, "Sensor Array Processing Based on Subspace Fitting", IEEE Trans. on ASSP (accepted for publication), 1990.

[16] A. Swindlehurst and T. Kailath, "A Performance Analysis of Subspace-Based Methods in the Presence of Model Errors - Part 2: Multidimensional Algorithms", IEEE Trans. on ASSP (in review), 1990.

[17] A. Swindlehurst and T. Kailath, "A Performance Analysis of Subspace-Based Methods in the Presence of Model Errors Part 1: The MUSIC Algorithm", IEEE Trans. on ASSP (in review), 1990 